\title{
Outbreak of carbapenem-resistant Providencia rettgeri in a tertiary hospital
}

\author{
V S Tshisevhe, ${ }^{1,2}$ MB ChB, MBA, Dip HIV Man (SA), DTM\&H; M R Lekalakala, ${ }^{3}$ BSc; MB ChB, DTM\&H, MMed, PDIC; \\ N Tshuma, ${ }^{4}$ BSc, MBA; S Janse van Rensburg, ${ }^{1}$ B Med Tech; N Mbelle, ${ }^{1,2}$ BSc, MSc, MB BCh, MMed, DTM\&H, MAP, FCPath (SA) \\ ${ }^{1}$ Department of Medical Microbiology, Tshwane Academic Division, National Health Laboratory Service, Pretoria, South Africa \\ ${ }^{2}$ Department of Medical Microbiology, Faculty of Health Sciences, University of Pretoria, South Africa \\ ${ }^{3}$ Department of Medical Microbiology, Polokwane National Health Laboratory Service, and Department of Medical Microbiology, \\ Faculty of Health Sciences, University of Limpopo, Polokwane, South Africa \\ ${ }^{4}$ Community AIDS Response, Johannesburg, South Africa
}

Corresponding author: VS Tshisevhe (vhudzani@gmail.com)

\begin{abstract}
The emergence of resistance to multiple antimicrobial agents in pathogenic bacteria is a significant public health threat, as there are limited effective antimicrobial agents for infections caused by multidrug-resistant (MDR) bacteria. Several MDR bacteria are now frequently detected. Carbapenem resistance in Enterobacteriaceae is often plasmid mediated, necessitating stringent infection control practices. We describe an outbreak of carbapenem-resistant Providencia rettgeri involving 4 patients admitted to intensive care and high-care units at a tertiary hospital. Clinical and demographic characteristics of 4 patients with carbapenem-resistant $P$. rettgeri were documented. All $P$. rettgeri isolated in these cases had a carbapenem-resistant antibiogram, with resistance to imipenem, ertapenem and meropenem. These cases could be epidemiologically linked. A multiprong approach, simultaneously targeting antibiotic stewardship, universal precautions and appropriate transmission-based precaution practices, is integral to prevention and control of nosocomial infections.
\end{abstract}

S Afr Med J 2017;107(1):31-33. DOI:10.7196/SAMJ.2017.v107i1.12002

Providencia rettgeri is a member of the Enterobacteriaceae, known to cause urinary tract infections (UTIs), bacteraemia, skin infections, gastroenteritis and wound infections, especially in immunocompromised patients and those with indwelling urinary catheters. ${ }^{[1-3]}$ P. rettgeri is an aerobic Gram-negative bacillus that displays marked resistance to most of the currently available antibiotics. ${ }^{[2]}$ The organism is part of the normal gastrointestinal flora. ${ }^{[3]}$

Immunocompromised or debilitated patients are highly susceptible to hospital-acquired infections - either after colonisation with environmental strains or following invasive procedures, such as catheterisation, bronchoscopy, colposcopy or surgical biopsies. ${ }^{[4]}$ P. rettgeri is commonly found to cause traveller's diarrhoea and has also been isolated from more severe human infections such as meningitis. ${ }^{[3,5]}$

Among the Providencia species, $P$. stuartii and $P$. rettgeri are the most common causes of infections, especially UTIs in hospitalised patients, although other infections can occur. Virtually all Providencia species can produce inducible AmpC beta-lactamases, and many isolates may also produce extended-spectrum beta-lactamases (ESBLs) in nosocomial settings. ${ }^{[3]}$

P. rettgeri has been associated with hospital-acquired infections, including catheter-related UTIs. Multiple factors contribute to the high frequency of UTIs in hospitalised patients. Comorbid illnesses and interventions to manage bladder voiding are the most important contributing variables. Prostatic hypertrophy in men promotes infection through urethral obstruction and turbulent urine flow and the additional risks associated with the use of instruments. Bacterial prostatitis, once established, is difficult to eradicate and may be a source of recurrent UTI over many years..$^{[7]}$

The emergence of resistance to multiple antimicrobial agents in pathogenic bacteria has become a significant public health threat, as there are fewer and sometimes no effective antimicrobial agents available for infections caused by multidrug-resistant bacteria. ${ }^{[8]}$

The mechanism of resistance of Enterobacteriaceae to carbapenems is most commonly by the production of an enzyme that breaks down the antibiotic before it can affect the bacterium. ${ }^{[9]}$ Resistance to antimicrobial agents in several bacteria is increasing, often because of poor application of antibiotic stewardship. Antimicrobial resistance is destroying the premise of modern medicine, i.e. whenever required, adequate antibiotic cover is available to save the patient's life. ${ }^{[10]}$ The emergence of carbapenem resistance in Providencia species is of particular concern because these bacteria are intrinsically resistant to polymyxins and tigecycline. ${ }^{[11]}$ These antibiotics are often used as part of regimens in the treatment of carbapenem-resistant isolates.

New Delhi metallo-beta-lactamase-1 (NDM-1)-mediated carbapenemase is the most common class B carbapenemase in Enterobacteriaceae and has been detected increasingly in several countries. ${ }^{[12]}$ The broad distribution of NDM-producing isolates and the apparent ease of transfer of the blaNDM gene are a major threat to public health globally. ${ }^{[13]}$ $P$. rettger $i$ has recently been recognised as a key organism for the dissemination of NDM-1, requiring measures for timely control of the spread of this organism. ${ }^{[14]}$ Our objective was to characterise a cluster of identified carbapenem-resistant $P$. rettger $i$ and determine the epidemiological link of the isolates.

\section{Methods}

A P. rettgeri outbreak was identified at Steve Biko Academic Hospital (SBAH), Pretoria, South Africa between November 2014 and January 2015. SBAH is a tertiary hospital with referrals from lower-level hospitals in the Tshwane municipal area. The urine samples were obtained from patients admitted to SBAH and the analysis was 
conducted at the medical microbiology laboratory of the Tshwane Academic Division of the National Health Laboratory Service (NHLS).

Ethical clearance was given by the Faculty of Health Sciences Research Ethics Committee, University of Pretoria (ref. no. 247/2015).

\section{Bacteria isolation and identification}

A loopfull of urine was streaked on blood agar and MacConkey agar media. The culture plates were incubated at $35 \pm 2^{\circ} \mathrm{C}$ for $18-24$ hours. Plates that showed growth were observed and the colonies were further identified using the Vitek 2 system (bioMérieux, USA).

\section{Antibiotic susceptibility test}

The following antibiotics were tested by the disc diffusion method: ampicillin $(10 \mu \mathrm{g})$, amoxicillin-clavulanic acid $(30 \mu \mathrm{g})$, cefotaxime $(30 \mu \mathrm{g})$, ceftazidime $(30 \mu \mathrm{g})$, cefoxitin $(30 \mu \mathrm{g})$, imipenem $(10 \mu \mathrm{g})$, meropenem $(10 \mu \mathrm{g})$, ertapenem $(10 \mu \mathrm{g})$, cefuroxime $(30 \mu \mathrm{g})$, ciprofloxacin $(5 \mu \mathrm{g})$, and trimethoprim/sulfamethoxazole $(25 \mu \mathrm{g})$. The isolates were inoculated on Mueller-Hinton agar and the antibiotic discs were placed on the agar surfaces. The plates were incubated at $35 \pm 2^{\circ} \mathrm{C}$ for 18 - 24 hours. The susceptibility was read after 24 hours' incubation as per 2015 Clinical and Laboratory Standards Institute guidelines.

Detection of ESBL production was determined among the Gramnegative bacteria resistant to third-generation cephalosporins by the double-disc synergy test, with ceftazidime $(30 \mu \mathrm{g})$ and cefotaxime $(30 \mu \mathrm{g})$ discs placed $20 \mathrm{~mm}$ centre-to-centre around an amoxicillin-clavulanic acid $(30 \mu \mathrm{g})$ disc. This was done on the surface of a Mueller-Hinton agar plate inoculated with the isolates' suspension equivalent to $0.5 \mathrm{McF}$ arland standards by surface spreading using sterile swab sticks. After incubation at $35 \pm 2^{\circ} \mathrm{C}$ for 24 hours, ESBL production was inferred when the zone of inhibition around the cephalosporin discs was expanded by $\geq 5 \mathrm{~mm}$ by the presence of clavulanic acid. Carbapenem resistance was defined by reduced susceptibility to imipenem, ertapenem and meropenem. Carbapenem resistance was characterised for carbapenemase production by the modified Hodge test.

\section{Resullts}

A total of 4 isolates were collected from patients who were admitted to the intensive care unit (ICU) and high-care unit. Clinical and demographic characteristics of the 4 patients with carbapenemresistant $P$. rettgeri are outlined in Table 1.

\section{Case 1}

An HIV-positive 26-year-old woman who was not yet receiving treatment was admitted to the ICU with a respiratory tract infection. While in the ICU, she developed renal failure and received dialysis. After 2 months she presented with pyrexia, haematuria and positive urine nitrites. A urine sample was submitted to the laboratory for culture, where $P$. rettgeri was isolated.

\section{Case 2}

An HIV-positive 32-year-old woman was admitted to the ICU a month after Case 1; they shared the ICU. She had renal failure with pulmonary oedema and received dialysis while there. A urine sample was submitted to the laboratory a month later for a suspected UTI; P. rettgeri was isolated. After 2 months in the ICU the patient was transferred to the high-care unit, where she continued receiving treatment for renal failure.

\section{Case 3}

A 40-year-old man with known hypertension was admitted to the same high-care unit as Case 2. He presented with renal failure, for which he received dialysis. Four days after admission he complained of symptoms suggestive of a possible UTI. A midstream urine sample was collected and sent to the laboratory, where P. rettgeri was isolated. The patient was deemed stable and transferred to a regular ward to continue with renal failure treatment.

\section{Case 4}

An HIV-positive 33-year-old woman was admitted to the surgical ICU with multiple gunshot wounds of the head and abdomen. The patient had a laparotomy, and a hysterectomy and nephrectomy were performed. Amoxicillin-clavulanic acid and metronidazole were administered after the operation to cover for possible contamination during surgery. While in the ICU, the patient developed bedsores on the sacral and occipital regions. Three weeks after admission, she was transferred to the high-care unit, where cases 2 and 3 were previously admitted. While in the high-care unit, a biopsy of the bedsore was sent to the laboratory, where $P$. rettgeri was isolated.

All $P$. rettgeri isolated in these cases had a carbapenem-resistant antibiogram with resistance to imipenem, ertapenem and meropenem.

\section{Discussion}

The cases presented in this study demonstrated carbapenem-resistant P. rettgeri, which showed that they were epidemiologically linked. This may partly be explained by some of the carbapenem-resistant genes being known to be transmitted horizontally. This organism inhabits moist areas or moisture-containing instruments, such as dialysis machines, which may have been the main source in this outbreak. As some patients acquired the infection after they were discharged, transmission from inanimate objects and healthcare workers is a possibility.

Several studies have indicated that immune-compromised patients are more prone to UTIs - all of the cases reported here were immune-

Table 1. Clinical and demographic characteristics of 4 cases of carbapenem-resistant $P$. rettgeri

\begin{tabular}{|c|c|c|c|c|c|c|c|c|}
\hline Case & Sex & $\begin{array}{l}\text { Age } \\
\text { (years) }\end{array}$ & Site of isolation & $\begin{array}{l}\text { Antimicrobial } \\
\text { therapy }\end{array}$ & Treatment & Outcome & HIV status & $\begin{array}{l}\text { Reason for ward } \\
\text { admission }\end{array}$ \\
\hline 1 & $\mathrm{~F}$ & 26 & Urine & Carbapenem & Dialysis & $\begin{array}{l}\text { Transferred to } \\
\text { high-care unit }\end{array}$ & Positive & $\begin{array}{l}\text { Respiratory } \\
\text { infection }\end{array}$ \\
\hline 2 & $\mathrm{~F}$ & 32 & Urine & Carbapenem & Dialysis & $\begin{array}{l}\text { Discharged } \\
\text { home }\end{array}$ & Positive & Renal failure \\
\hline 3 & M & 40 & Urine & Carbapenem & Dialysis & $\begin{array}{l}\text { Transferred to } \\
\text { renal unit }\end{array}$ & Negative & Renal failure \\
\hline 4 & $\mathrm{~F}$ & 33 & Tissue & Carbapenem & Dialysis & Died & Positive & Polytrauma \\
\hline
\end{tabular}


compromised. ${ }^{[1-3]}$ Catheterisation is a predisposing factor for the development of a UTI and all patients investigated in this study had urinary catheterisation. All patients were also dialysed, which may have exposed them to fluids containing $P$. rettgeri. This association will require a larger study to provide conclusive evidence.

Caution needs to be exercised when using common equipment, such as dialysis machines, to prevent the transfer of bacteria. Thorough sterilisation and cleaning of equipment is of paramount importance in the prevention of microbial contamination and infection. A point prevalence surveillance of colonisation must be conducted on a regular basis for early detection of environmental and equipment contamination. Cohorting of patients infected and colonised by resistant organisms may limit the spread of infection to other patients in the unit. Studies have demonstrated that isolating and cohorting infected or colonised patients with contact precautions, healthcare personnel hand hygiene and environmental cleaning are integral to the disruption of the spread of infection within the hospital setting. ${ }^{[15]}$

\section{Conclusion}

The prevention and control of nosocomial infections depend on a multipronged approach, simultaneously targeting antibiotic stewardship, universal precautions and appropriate transmission-based precaution practices. Education of healthcare personnel plays a pivotal role in preventing and controlling the outbreak. Hand hygiene and equipment sterilisation must be reiterated to healthcare personnel, as proper hygienic practices are important in the prevention of bacterial infections in a healthcare facility.
1. Cho H-J, Lim S-J, Chun S-Y, et al. A case of Providencia rettgeri sepsis in a patient with cervical cord injury. Infect Chemother 2010:42(6):428. http://dx doi org/10.3947/ic 2010.42.6.428

2. Olaitan AO, Diene SM, Assous MV, Rolain JM. Genomic plasticity of multidrug-resistant NDM-1 positive . Olaitan AO, Diene SM, Assous MV, Rolain JM. Genomic plasticity of multidrug-resistant NDM-1 positive
clinical isolate of Providencia rettgeri. Genome Biol Evol 2016;8(3):723-728. http://dx.doi.org/10.1093/gbe/ evv195

3. Zavascki AP, Carvalhaes CG, da Silva GL, et al. Outbreak of carbapenem-resistant Providencia stuartii in an intensive care unit. Infect Control Hosp Epidemiol 2012;33(6):627-630. http://dx.doi.org/10.1086/665730

4. Pollett S, Miller S, Hindler J, Uslan D, Carvalho M, Humphries RM. Phenotypic and molecular characteristic of carbapenem-resistant Enterobacteriaceae in a health care system in Los Angeles, California, from 2011 to 2013. J Clin Microbiol 2014;52(11):4003-4009. http://dx.doi.org/10.1128/JCM.01397-14

5. Galac MR, Lazzaro BP. Comparative genomics of bacteria in the genus Providencia isolated from wild Drosophila melanogaster. BMC Genomics 2012;13(1):612. http://dx.doi.org/10.1186/1471-2164-13-612

6. Barrios H, Garza-Ramos U, Reyna-Flores F, Sanchez-Perez A, Rojas-Moreno T, Garza-Gonzalez E Isolation of carbapenem-resistant NDM-1-positive Providencia rettgeri in Mexico. J Antimicrob Chemother 2013;68(8):1934-1936. http://dx.doi.org/10.1093/jac/dkt124

7. Nicolle LE. Urinary tract infections in long-term-care facilities. Infect Control Hosp Epidemiol 2001;22(3):167-175. http://dx.doi.org/10.1086/501886

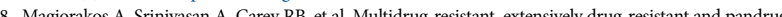
Magiorakos A, Srinivasan A, Carey RB, et al. Multidrug-resistant, extensively drug-resistant and pandrug resistant bacteria: An international expert proposal for interim standard definitions for acquire
Clin Microbiol Infect 2011;18(3):268-281. http://dx.doi.org/10.1111/j.1469-0691.2011.03570.x

Clin Microbiol Infect 2011;18(3):268-281. http://dx.doi.org/10.1111/j.1469-0691.2011.03570.x 9ustralian Commission on Safety and Quality in Health Care. Recommendations for the control of
multi-drug resistant Gram-negatives: Carbapenem resistant Enterobacteriaceae. 2013. http://www multi-drug resistant Gram-negatives: Carbapenem resistant Enterobacteriaceae. 2013. http://wWW-
safetyandquality.gov.au/wp-content/uploads/2013/12/MRGN-Guide-Enterobacteriaceae-PDF-1.89MB safetyandquality.gov.au/wp-conte
pdf (accessed 4 November 2016)

0. Rodrigues C. Carbapenem-resistant Enterobacteriaceae: A reality check. Regional Health Forum 2011;15(1):83-86.

11. Sidjabat H, Nimmo GR, Walsh TR, et al. Carbapenem resistance in Klebsiella pneumoniae due to the New Delhi metallo- $\beta$-lactamase. Clin Infect Dis 2011;52(4):481-484. http://dx.doi.org/10.1093/cid/ciq178

12. Rozales FP, Ribeiro VB, Magagnin CM, et al. Emergence of NDM-1-producing Enterobacteriaceae in Porto Alegre, Brazil. Int J Infect Dis 2014;25:79-81. http://dx.doi.org/10.1016/j.ijid.2014.01.005

13. Pasteran F, Meo A, Gomez S, et al. Emergence of genetically related NDM-1-producing Providencia rettgeri strains in Argentina. J Glob Antimicrob Resist 2014;2(4):344-345. http://dx.doi.org/10.1016/j jgar.2014.07.003

14. Gefen-Halevi S, Hindiyeh MY, Ben-David D, et al. Isolation of genetically unrelated bla(NDM-1)-positive Providencia rettgeri strains in Israel. J Clin Microbiol 2013;51(5):1642-1643. http://dx.doi.org/10.1128 JCM.00381-13

15. Maltezou HC. Metallo-beta-lactamases in Gram-negative bacteria: Introducing the era of panresistance? Int J Antimicrob Agents 2009;33(5):405. http://dx.doi.org/10.1016/j.ijantimicag.2008.09.003

Accepted 19 September 2016. 